\title{
High Doses of PDE5 inhibitors and tramadol reversibly alters haematological parameters in rats
}

\author{
Victor U. Nna*, Victor O. Oka, Augustine L. Udefa, Emmanuel O. Ofutet, Ofem E. Ofem \\ Department of Physiology, Faculty of Basic Medical Sciences, College of Medical Sciences, University of Calabar, P.M.B. 1115 Calabar, \\ Cross River State, Nigeria.
}

\begin{tabular}{l} 
ARTICLE INFO \\
\hline Article history: \\
Received on: 11/02/2016 \\
Revised on: 18/03/2016 \\
Accepted on: 02/04/2016 \\
Available online: 30/04/2016 \\
\hline Key words: \\
Blood cell count, PDE5, \\
sildenafil, tadalafil, tramadol, \\
platelets.
\end{tabular}

platelets.

\begin{abstract}
This study examined the effect of chronic administration of PDE5 inhibitors and tramadol on haematological indices because of their reported high incidence of abuse. Additionally, the possibility of reversal of negative effects following withdrawal of treatment was examined. Fifty male rats $(180-200 \mathrm{~g}$ body weight $)$ were grouped into five $(\mathrm{n}=10)$, namely: control, sildenafil, tadalafil, tramadol and sildenafil+tramadol group. The different groups were orally treated with $0.2 \mathrm{~mL}$ normal saline, sildenafil $(1 \mathrm{mg} / 100 \mathrm{gb} . \mathrm{w}$.$) , tadalafil (1 \mathrm{mg} / 100 \mathrm{gb} . \mathrm{w}$.), tramadol ( $2 \mathrm{mg} / 100 \mathrm{~g}$ b.w. $)$ and sildenafil + tramadol $(1 \& 2 \mathrm{mg} / 100 \mathrm{gb} . \mathrm{w}$. respectively). Treatment was done thrice a week, for 8 weeks and the animals were allowed access to feed and water ad libitum. Five animals were sacrificed per group, while the remaining 5/group continued for another 8 weeks without drug administration (recovery test).Blood samples were collected from each animal via cardiac puncture at the end of both phases for assessment of haematological parameters. Red blood cells (RBC) count, haemoglobin $(\mathrm{Hb})$ concentration, packed cell volume (PCV), mean corpuscular volume (MCV), mean corpuscular haemoglobin ( $\mathrm{MCH})$, mean corpuscular haemoglobin concentration (MCHC), red cell distribution wide standard deviation (RDW-SD), white blood cells (WBCs) count, platelets count, mean platelets volume (MPV) and platelets large cell ratio (PLCR) were significantly reduced in all the treated groups compared with the control. Following withdrawal of treatment, $\mathrm{RBC}$ count, $\mathrm{Hb}$ concentration, $\mathrm{PCV}$ and red cell absolute values were significantly increased in all recovery groups compared with their respective treated groups. Haematological alterations were reversed following withdrawal of treatment. However, platelet indices were poorly reversed in sildenafil and tramadol recovery groups.
\end{abstract}

\section{INTRODUCTION}

Phosphodiesterase type 5 inhibitors (PDE5i) are drugs used to treat erectile dysfunction of various etiologies in men. PDE5i are also used in the treatment of pulmonary hypertension (Supuran et al., 2006; Aversa et al., 2006). These drugs act selectively in the smooth muscle of the lungs and penis because their receptors are primarily distributed within these areas (Oka et al., 2015). PDE5i are so called because they selectively inhibit the action of phosphodiesterase type 5, an enzyme which

\footnotetext{
* Corresponding Author

Victor U. Nna, Department of Physiology, Faculty of Basic Medical Sciences, College of Medical Sciences, University of Calabar, P.M.B. 1115 Calabar, Cross River State, Nigeria.

Email:victor2nna[at]gmail.com
}

promotes the degradation of cyclic guanosine monophosphate (cGMP) (Salam et al., 2015).Thus, erection is prolonged as long ascGMP is not degraded. Examples of PDE5i are sildenafil citrate (common name: Viagra) and Tadalafil (common name: Cialis). Both sildenafil and tadalafil are known to affect the body in different ways. The effect of sildenafil has been widely examined in different body systems including respiratory (Goldberg et al., 2011), renal (Lauver et al., 2014), gastrointestinal (Eherer et al., 2002), nervous (Kyratsas et al., 2013) and cardiovascular (Zusman et al., 2000; Shinlapawittayatorn et al., 2005) systems. Haematological studies with sildenafil by Yildiz et al. (2011) showed that sildenafil administration at low dose significantly increased the levels of red blood cells (RBC) GSH and lymphocyte count but decreased the levels of MDA in plasma and RBC, blood mean corpuscular volume and eosinophil counts. 
At higher doses, sildenafil has shown to significantly increase the levels of GSH in RBC, and blood lymphocyte counts, but decreased plasma RBC and MDA levels. Another study by Tas et al. (2011) on sildenafil's effect on haematological parameters in early phase of wound healing in diabetic rats revealed that, sildenafil played an important role in relieving the pressure on cellular activities and maintaining the wound healing within normal limits. Their findings showed that sildenafil administered to diabetic rats increased neutrophil and monocytes counts. Shatha and Adnan (2015) in their study on blood homeostasis concluded that PDE5i (sildenafil and tadalafil) increased platelets activity and activated their aggregation. Their findings also showed that sildenafil had less aggregatory effects on platelets than tadalafil when used for the same duration of time. Nna et al. (2015a) reported that sildenafil and tadalafil administered separately exhibited hyperlipidemic effects in male rats.

Tramadol is a centrally acting analgesic agent with activity at $\mu$-opioid, adrenergic and 5-hydroxytryptamine (5-HT) receptors. It acts in two ways: by (1) binding of parent and M1 metabolite to $\mu$ opioid receptors in animals and (2) weak inhibition of re-uptake of nor-epinephrine and serotonin (Bamigbade et al., 1997; Grond and Sablotzki, 2004). This is the basis for which tramadol is used in the treatment of premature ejaculation (Safarinejad and Hosseini, 2006; Salem et al., 2008). Tramadol has been reported to decrease cortisol concentration (Oka et al., 2015), and increase basal metabolic rate and triiodothyronine concentration (Oka et al., 2015). Tramadol has also been reported to significantly alter liver functions (Nna et al., 2015b). In haematological studies conducted by Elyazji et al. (2013), tramadol was found to increase WBC count, lymphocyte count and $\mathrm{MCV}$, but decreased PCV, $\mathrm{Hb}, \mathrm{RBC}$ count, $\mathrm{MCH}, \mathrm{MCHC}$ and platelets count. Their findings showed signs of improvement of blood indices in the recovery periods after tramadol abstinence. Another study by Akhtardanesh et al. (2014) in dogs showed that short-term injection of high doses of tramadol did not change haematological parameters significantly.

Recent studies have shown that some users combine sildenafil and tramadol (Aldalou et al. 2014; Oka et al., 2015) in a bid to prolong erection and increase their genital size (Nna et al., 2014a,b). In our previous study, combined administration of sildenafil and tramadol was shown to significantly alter liver functions but these alterations were reversed following withdrawal of treatment with the drugs (Nna et al., 2015b). Our findings also revealed that, combination of sildenafil and tramadol exhibited hypolipidemic effect with poor reversibility following withdrawal of treatment. Aldalou et al. (2014) reported that combined administration of sildenafil and tramadol significantly increased WBCs count, MCV, $\mathrm{MCH}$ and $\mathrm{MCHC}$, but significantly decreased RBCs count, platelet countand $\mathrm{Hb}$ concentration in domestic rabbits. Despite the vast research done with sildenafil, tadalafil and tramadol, separately and in combination, there is little or no report on whether the alterations caused by these drugs on haematological parameters can be reversed if treatment is withdrawn. The present study therefore seeks to evaluate possible reversal of haematological alterations in rats following treatment and subsequent withdrawal of treatment with PDE5i (sildenafil and tadalafil) and opioid (tramadol) separately and in combination.

\section{MATERIALS AND METHODS}

\section{Animal preparation and protocol}

The study utilized fifty (50) male albino wistar rats (180 - 200g body weight) bought from Department of Agriculture, University of Calabar. The animals were handled according to Helsinki's (1964) laid down principles. They were kept in properly ventilated wooden cages in the animal house of Physiology Department, University of Calabar, allowed access to rat feed and water ad libitum and exposed to 12/12 hours light/dark cycle. The animals were allowed for seven (7) days to explore their new environment before commencement of treatment.

\section{Experimental design and drug administration}

The 50 rats were randomly assigned into five (5) groups $(n=10)$ thus: control, sildenafil, tadalafil, tramadol and sildenafilt+ tramadol $(\mathrm{S}+\mathrm{T})$ groups. The drugs were purchased from BEZ Pharmacy, Etta Agbor road, Calabar, Nigeria and administered per oral route, once, every two days, for 8 weeks. The doses administered were as previously used (Nna et al., 2015a, b). All groups had accessed to rat feed and distilled water. Furthermore, sildenafil group received sildenafil citrate (Maxheal Laboratories Pvt Ltd, India) at $1 \mathrm{mg} / 100 \mathrm{~g}$ b.w., tadalafil group received tadalafil ( (Pfizer, India) at $1 \mathrm{mg} / 100 \mathrm{~g}$ b.w., tramadol group was given tramadol hydrochloride (Glow PharmaPvt Ltd, India) at $2 \mathrm{mg} / 100 \mathrm{~g}$ b.w., while the sildenafil+tramadol group received both sildenafil and tramadol at $1 \mathrm{mg} / 100 \mathrm{~g} \mathrm{~b} . \mathrm{w}$ and $2 \mathrm{mg} / 100 \mathrm{~g} \mathrm{~b}$.w, respectively. At the end of 8 weeks of treatment, five animals from each group were randomly selected and sacrificed. Blood samples were collected for assessment of haematological parameters. The remaining five (5) animals per group were allowed for another 8 weeks, without treatment (recovery phase), but were allowed access to feed and water ad libitum. They were then sacrificed at the sixteenth week and blood samples collected for assessment of blood parameters.

\section{Collection of blood sample}

At the end of the first and second phases, animals were sacrificed under chloroform anaesthesia (3.8\%). Collection of blood samples was done using $5 \mathrm{ml}$ syringes with $21 \mathrm{G}$ needles. The samples were collected from the animals through cardiac puncture into pre-labelled ethylenediaminetetracetate (EDTA) vials and gently agitated to ensure EDTAis spread uniformly after which the samples were immediately used for measurement of haematological parameters.

\section{Measurement of haematological parameters}

Haematological parameters were measured using automated cell counter (Coulter Electronics, Luton, Bedfordshine, 
$\mathrm{UK}$ ) having standard calibrations in line with the instructions of the manufacturer. Parameters measured were: RBC count, WBC count, platelet count, lymphocyte count, PCV, Hb concentration, MCV, MCH, MCHC, PDW, MPV, P-LCR, RDW-SD and RDW$\mathrm{CV}$.

\section{Statistical analysis}

Results are presented as mean \pm standard error of mean (SEM). Computer software, SPSS (version 17.0) was used for data analysis. Statistical measures used were one way analysis of variance (ANOVA) along with post hoc multiple comparison test (least square difference procedure). Values ofp $<0.05$ was the criterion for statistical significance.

\section{RESULTS}

\section{Comparison of haematological parameters in the different experimental groups following 8 weeks of treatment with the various drugs \\ Red blood cell, Haemoglobin concentration and Packed cell volume}

Table 1 shows the RBC count $\left(\times 10^{6}\right.$ cell $\left./ \mu \mathrm{L}\right)$ for control, sildenafil, tadalafil, tramadol and $\mathrm{S}+\mathrm{T}$ groups. RBC count was significantly $(\mathrm{p}<0.01)$ lower in the sildenafil and tadalafil groups compared with control group. It was also significantly $(\mathrm{p}<0.001)$ lower in tramadol and $\mathrm{S}+\mathrm{T}$ groups compared with control. Sildenafil and tadalafil groups presented a significantly $(\mathrm{p}<0.05$; $\mathrm{p}<0.01$ respectively) higher $\mathrm{RBCs}$ count compared with $\mathrm{S}+\mathrm{T}$ group. The haemoglobin $(\mathrm{Hb})$ concentration $(\mathrm{g} / \mathrm{dL})$ for all the groups is also shown in table 1 . Hb concentration was significantly $(\mathrm{p}<0.001)$ lower in sildenafil, tramadol and $\mathrm{S}+\mathrm{T}$ groups compared with control. The tramadol and $\mathrm{S}+\mathrm{T}$ groups presented a significant $(\mathrm{p}<0.001)$ decrease in $\mathrm{Hb}$ concentration compared with tadalafil group. $\mathrm{Hb}$ concentration was significantly $(\mathrm{p}<0.001)$ higher in sildenafil group compared with tadalafil group. PCV (\%) was significantly $(\mathrm{p}<0.001)$ lower in sildenafil, tramadol and $\mathrm{S}+\mathrm{T}$ groups compared with control. It was also significantly $(\mathrm{p}<0.05)$ lower in tadalafil group compared with control. The tramadol group presented a significant $(\mathrm{p}<0.05)$ decrease in PCV compared with tadalafil group. PCV was significantly $(\mathrm{p}<0.05)$ higher in sildenafil group compared with tadalafil group (Table 1).

\section{Red blood cell absolute values}

Table 2 shows the red blood cells absolute values for control, sildenafil, tadalafil, tramadol and $\mathrm{S}+\mathrm{T}$ groups. The sildenafil, tramadol and $\mathrm{S}+\mathrm{T}$ groups presented a significant $(\mathrm{p}<0.001)$ decrease in MCV (fL) compared with control group. MCV was significantly $(\mathrm{p}<0.001)$ lower in tramadol and $\mathrm{S}+\mathrm{T}$ groups compared with sildenafil and tadalafil groups. The tadalafil group presented a significant $(\mathrm{p}<0.001)$ increase in $\mathrm{MCV}$ compared with sildenafil group and a significant $(\mathrm{p}<0.05)$ decrease compared with control group. All the treated groups presented a significant $(\mathrm{p}<0.001)$ decrease in $\mathrm{MCH}(\mathrm{pg})$ concentration compared with control. The tadalafil group presented a significant $(\mathrm{p}<0.001)$ decrease in $\mathrm{MCH}$ compared with sildenafil group. $\mathrm{MCH}$ was significantly $(\mathrm{p}<0.001)$ lower in tramadol and $\mathrm{S}+\mathrm{T}$ groups compared with tadalafil group. MCHC (g/dL) was significantly $(\mathrm{p}<0.001)$ lower in all treated groups compared with control. Sildenafil, tramadol and $\mathrm{S}+\mathrm{T}$ groups presented a significant $(\mathrm{p}<0.001)$ decrease in $\mathrm{MCHC}$ compared with tadalafil group. MCHC was significantly $(\mathrm{p}<0.05)$ lower in $\mathrm{S}+\mathrm{T}$ groups compared with sildenafil group. RDW-SD (fL) was significantly $(\mathrm{p}<0.01)$ lower in sildenafil and tadalafil groups compared with control. The tramadol and $\mathrm{S}+\mathrm{T}$ groups also presented a significant $(\mathrm{p}<0.001)$ decrease in RDW-SD compared with control.

RDW-SD was significantly $(\mathrm{p}<0.001)$ decreased in sildenafil, tadalafil and tramadol groups compared with $\mathrm{S}+\mathrm{T}$. The tramadol group presented a significant $(\mathrm{p}<0.05 ; \mathrm{p}<0.01)$ decrease in RDW-SD compared with sildenafil and tadalafil groups respectively. $\mathrm{S}+\mathrm{T}$ group had a significantly $(\mathrm{p}<0.001)$ lower RDW-CV (\%) compared with control. RDW-CV was significantly $(\mathrm{p}<0.01)$ higher in sildenafil, tadalafil and tramadol groups compared with $\mathrm{S}+\mathrm{T}$.

Table 1: Comparison of RBC, Hb concentration and PCV in the different experimental groups after 8 weeks of treatment.

\begin{tabular}{|c|c|c|c|}
\hline Groups (Batch A) & $\mathrm{RBC}\left(\mathrm{x10}^{6}\right.$ cell $\left./ \mu \mathrm{L}\right)$ & $\mathrm{Hb}(\mathrm{g} / \mathrm{dL})$ & PCV (\%) \\
\hline Control & $7.32 \pm 0.24$ & $14.20 \pm 0.50$ & $44.40 \pm 1.41$ \\
\hline Sildenafil & $5.55 \pm 0.49^{\mathbf{b}, \mathbf{z}}$ & $10.57 \pm 0.43^{\mathbf{a}, \mathbf{d}}$ & $37.74 \pm 0.46^{\mathrm{a}, \mathrm{f}}$ \\
\hline Tadalafil & $5.67 \pm 0.57^{\mathbf{b}, \mathbf{y}}$ & $13.94 \pm 0.21$ & $41.48 \pm 0.66^{\mathrm{c}}$ \\
\hline Tramadol & $4.80 \pm 0.19^{\mathrm{a}}$ & $10.52 \pm 0.46^{\mathbf{a , d}}$ & $38.38 \pm 0.39^{\mathbf{a}, \mathbf{f}}$ \\
\hline$S+T$ & $3.95 \pm 0.07^{\mathrm{a}}$ & $9.76 \pm 0.89^{\mathbf{a , d}}$ & $35.56 \pm 1.04^{\mathrm{a}}$ \\
\hline
\end{tabular}

Table 2: Comparison of red blood cell absolute values (MCV, MCH, MCHC, RDW-SD and RDW-CV) in the different groups after 8 weeks of treatment.

\begin{tabular}{|c|c|c|c|c|c|}
\hline Groups (Batch A) & MCV (fL) & MCH (pg) & $\operatorname{MCHC~(g/dL)~}$ & RDW-SD (fL) & RDW-CV (\%) \\
\hline Control & $61.44 \pm 1.26$ & $19.62 \pm 0.37$ & $31.68 \pm 0.22$ & $33.56 \pm 0.76$ & $13.82 \pm 0.68$ \\
\hline Sildenafil & $51.16 \pm 1.02^{\mathrm{a}}$ & $11.06 \pm 0.45^{\mathrm{a}}$ & $21.92 \pm 0.25^{\mathrm{agg}}$ & $29.96 \pm 0.49^{\mathbf{b}, \mathbf{x}}$ & $12.24 \pm 0.46^{\mathbf{y}}$ \\
\hline Tadalafil & $57.92 \pm 0.69^{\mathbf{c}, \mathbf{d}}$ & $13.74 \pm 0.45^{\mathbf{a}, \mathbf{d}}$ & $25.66 \pm 0.71^{\mathrm{a}}$ & $30.22 \pm 0.82^{\mathbf{b , x}}$ & $12.72 \pm 0.69^{\mathbf{y}}$ \\
\hline Tramadol & $41.60 \pm 0.90^{\mathbf{a}, \mathbf{d}, \mathbf{g}}$ & $10.20 \pm 0.47^{\mathbf{a}, \mathrm{g}}$ & $21.52 \pm 0.74^{\mathrm{a}, \mathrm{g}}$ & $26.34 \pm 0.77^{\mathbf{a , f}, \mathbf{h}, \mathbf{x}}$ & $12.62 \pm 0.37^{\mathbf{y}}$ \\
\hline $\mathbf{S}+\mathbf{T}$ & $40.34 \pm 0.89^{\mathbf{a}, \mathbf{d}, \mathbf{g}}$ & $10.56 \pm 0.52^{\mathrm{a}, \mathrm{g}}$ & $19.88 \pm 0.42^{\mathbf{a g}, \mathbf{f}}$ & $21.42 \pm 0.77^{\mathbf{a}}$ & $9.80 \pm 0.12^{\mathrm{a}}$ \\
\hline
\end{tabular}

vstadalafil; $\mathrm{x}=\mathrm{p}<0.001, \mathrm{y}=\mathrm{p}<0.01$ vs $\mathrm{S}+\mathrm{T}$. 
Table 3: Comparison of WBC counts and Platelet indices in the different experimental groups after 8 weeks of treatment.

\begin{tabular}{|c|c|c|c|c|c|c|}
\hline $\begin{array}{l}\text { Groups } \\
\text { (Batch A) }\end{array}$ & $\begin{array}{c}\mathrm{WBC} \\
\left(\mathbf{x 1 0} 0^{3} \mathrm{cell} / \mu \mathrm{L}\right)\end{array}$ & Lymphocytes (\%) & $\begin{array}{c}\text { Platelet } \\
\left(\mathrm{x} 10^{3} \text { cell/ } / \mu \mathrm{L}\right)\end{array}$ & $\begin{array}{c}\text { PDW } \\
(\mathbf{f L})\end{array}$ & $\begin{array}{r}\text { MPV } \\
(\mathbf{f L})\end{array}$ & P-LCR (\%) \\
\hline Control & $11.74 \pm 0.76$ & $74.68 \pm 2.69$ & $936.00 \pm 14.55$ & $11.50 \pm 0.80$ & $9.10 \pm 0.41$ & $18.06 \pm 2.89$ \\
\hline Sildenafil & $5.30 \pm 0.46^{\mathrm{a}}$ & $71.00 \pm 0.49^{\mathbf{z}}$ & $700.40 \pm 7.83^{\mathrm{a}}$ & $9.92 \pm 0.44^{\mathfrak{c}}$ & $8.16 \pm 0.17^{\mathfrak{c}}$ & $12.44 \pm 1.28^{\mathrm{c}}$ \\
\hline Tadalafil & $7.93 \pm 0.38^{\mathbf{a , e}}$ & $79.74 \pm 4.04^{\mathbf{f}, \mathbf{x}}$ & $686.60 \pm 15.13^{\mathrm{a}, \mathrm{f}}$ & $10.28 \pm 0.33$ & $8.26 \pm 0.15^{\mathrm{c}}$ & $11.88 \pm 0.58^{\mathrm{c}}$ \\
\hline Tramadol & $8.52 \pm 0.26^{\mathbf{a}, \mathbf{d}, \mathbf{z}}$ & $72.64 \pm 1.05^{\mathrm{y}}$ & $762.20 \pm 33.55^{\mathbf{a}, \mathbf{f}}$ & $9.44 \pm 0.07^{\mathbf{b}}$ & $7.76 \pm 0.12^{\mathrm{a}}$ & $10.16 \pm 0.75^{\mathbf{b}}$ \\
\hline $\mathbf{S}+\mathbf{T}$ & $6.50 \pm 0.32^{\mathrm{a}}$ & $62.48 \pm 0.61^{\mathbf{b}, \mathbf{f}}$ & $713.60 \pm 0.83^{\mathrm{a}}$ & $9.86 \pm 0.18^{\mathrm{c}}$ & $8.18 \pm 0.07^{\mathfrak{c}}$ & $12.66 \pm 0.57^{\mathrm{c}}$ \\
\hline \multicolumn{7}{|c|}{$\begin{array}{l}\text { Values are expressed as mean } \pm S E M, n=5 ., a=p<0.001, b=p<0.01, c=p<0.05 \text { vs control; } d=p<0.001, e=p<0.01, f=p<0.05 \text { vs sildenafil; } x=p<0.001, y= \\
p<0.01, z=p<0.05 \text { vs } S+T \text {. }\end{array}$} \\
\hline \multicolumn{2}{|l|}{ Groups (Batch B) } & $\mathrm{RBC}\left(\mathrm{x10} \mathbf{c}^{6}\right.$ cell$\left./ \mu \mathrm{L}\right)$ & \multicolumn{2}{|c|}{ Hb (g/dL) } & \multicolumn{2}{|c|}{ PCV (\%) } \\
\hline \multicolumn{2}{|l|}{ Control } & $7.71 \pm 0.16$ & \multicolumn{2}{|c|}{$14.74 \pm 0.17$} & \multicolumn{2}{|c|}{$45.58 \pm 0.81$} \\
\hline \multicolumn{2}{|l|}{ Sildenafil } & $7.86 \pm 0.17$ & \multicolumn{2}{|c|}{$14.80 \pm 0.28$} & \multicolumn{2}{|c|}{$46.70 \pm 1.32$} \\
\hline \multicolumn{2}{|l|}{ Tadalafil } & $7.30 \pm 0.27$ & \multicolumn{2}{|c|}{$13.84 \pm 0.33$} & \multicolumn{2}{|c|}{$44.40 \pm 1.09$} \\
\hline \multicolumn{2}{|l|}{ Tramadol } & $7.35 \pm 0.34$ & \multicolumn{2}{|c|}{$13.66 \pm 0.65$} & \multicolumn{2}{|c|}{$43.14 \pm 1.09$} \\
\hline \multicolumn{2}{|l|}{$\mathbf{S}+\mathbf{T}$} & $7.63 \pm 0.05$ & \multicolumn{2}{|c|}{$14.46 \pm 0.70$} & \multicolumn{2}{|c|}{$44.34 \pm 0.81$} \\
\hline
\end{tabular}

Values are expressed as mean \pm SEM, $n=5$.

Table 5: Comparison of red blood cell absolute values (MCV, MCH, MCHC, RDW-SD and RDW-CV) in the different groups after withdrawal of treatment.

\begin{tabular}{lccccc}
\hline Groups (Batch B) & MCV (fL) & MCH (pg) & MCHC (g/dL) & RDW-SD (fL) & RDW-CV (\%) \\
\hline Control & $61.36 \pm 0.99$ & $20.02 \pm 0.68$ & $31.86 \pm 0.58$ & $34.00 \pm 0.53$ & $14.72 \pm 0.86$ \\
Sildenafil & $59.36 \pm 0.56$ & $18.84 \pm 0.13$ & $31.72 \pm 0.34$ & $33.46 \pm 0.55$ & $15.16 \pm 0.46$ \\
Tadalafil & $58.16 \pm 0.65^{\mathbf{c}}$ & $17.96 \pm 0.33^{\mathbf{b}}$ & $31.56 \pm 0.50$ & $34.80 \pm 0.71$ & $16.08 \pm 0.69$ \\
Tramadol & $58.76 \pm 0.80^{\mathbf{c}}$ & $18.60 \pm 0.22^{\mathbf{c}}$ & $31.62 \pm 0.16$ & $36.48 \pm 2.26$ \\
S + T & $59.06 \pm 0.79$ & $18.34 \pm 0.20^{\mathbf{b}}$ & $30.68 \pm 0.47$ & $35.08 \pm 1.48$ & $17.14 \pm 1.33$ \\
\hline
\end{tabular}

Values are expressed as mean \pm SEM, $\mathrm{n}=5$.

$\mathrm{b}=\mathrm{p}<0.01, \mathrm{c}=\mathrm{p}<0.05$ vs control.

\section{WBC counts and platelet indices}

Results for WBC count $\left(\times 10^{3}\right.$ cell $\left./ \mu \mathrm{L}\right)$ and platelet indices are shown in table 3. All treated groups had a significantly $(p<0.001)$ lower WBC count compared with control. The tadalafil and tramadol groups presented a significant $(\mathrm{p}<0.01 ; \mathrm{p}<0.001$ respectively) increase in WBC count compared with sildenafil group. WBC count for tramado group was significantly $(\mathrm{p}<0.05)$ higher compared with $\mathrm{S}+\mathrm{T}$ group.

Lymphocytes count $(\%)$ was significantly $(\mathrm{p}<0.01)$ lower in $\mathrm{S}+\mathrm{T}$ group compared with control but significantly higher in sildenafil $(\mathrm{p}<0.05)$, tadalafil $(\mathrm{p}<0.001)$ and tramadol $(\mathrm{p}<0.01)$ groups compared with $\mathrm{S}+\mathrm{T}$.

Lymphocytes count was significantly $(\mathrm{p}<0.05)$ higher in tadalafil group and significantly $(\mathrm{p}<0.05)$ lower in $\mathrm{S}+\mathrm{T}$ group compared with sildenafil group. All the treated groups presented a significantly $(\mathrm{p}<0.001)$ lower platelet count $\left(\mathrm{x} 10^{3} \mathrm{cell} / \mu \mathrm{L}\right)$ compared with control. Tadalafil and tramadol groups had a significantly $(\mathrm{p}<0.05)$ lower platelet count compared with sildenafil group. PDW ( $\mathrm{fL}$ ) was significantly $(\mathrm{p}<0.05)$ lower in sildenafil and $\mathrm{S}+\mathrm{T}$ groups compared with control. PDW was also significantly $(\mathrm{p}<0.01)$ lower in tramadol group compared with control. MPV (fL) results show that sildenafil, tadalafil and $\mathrm{S}+\mathrm{T}$ groups presented a significantly $(\mathrm{p}<0.05)$ lower MPV compared with control. MPV was also significantly $(\mathrm{p}<0.001)$ lower in tramadol group compared with control.
P-LCR $(\%)$ was significantly $(\mathrm{p}<0.05)$ lower in sildenafil, tadalafil and $\mathrm{S}+\mathrm{T}$ groups compared with control. P-LCR was also significantly $(\mathrm{p}<0.01)$ lower in the tramadol group compared with control.

\section{Comparison of haematological parameters in the different experimental groups after 8 weeks of withdrawal of treatment}

\section{Red blood cell, Haemoglobin concentration and Packed cell volume}

Table 4 shows the results for RBCs count $\left(\times 10^{6}\right.$ cell $\left./ \mu \mathrm{L}\right)$, haemoglobin concentration $(\mathrm{g} / \mathrm{dL})$ and packed cell volume (\%) for control, sildenafil, tadalafil, tramadol and $\mathrm{S}+\mathrm{T}$ groups after withdrawal of treatment. There was no significant difference in RBCs count, haemoglobin concentration and packed cell volume in the different experimental groups.

\section{Red blood cell absolute values}

Results for red blood cells absolute values after withdrawal of treatment are presented in table 5. MCV (fL) was significantly $(\mathrm{p}<0.05)$ decreased in tadalafil and tramadol groups compared with control. $\mathrm{MCH}(\mathrm{pg})$ was significantly decreased in tadalafil ( $\mathrm{p}<0.01)$, tramadol $(\mathrm{p}<0.05)$ and $\mathrm{S}+\mathrm{T}(\mathrm{p}<0.01)$ groups compared with control. There was no significant difference in MCHC (g/dL), RDW-SD (fL) and RDW-CV (\%) in all the groups. 
Table 6: Comparison of WBC counts and Platelet indices in the different experimental groups after withdrawal of treatment.

\begin{tabular}{|c|c|c|c|c|c|c|}
\hline $\begin{array}{c}\text { Groups } \\
\text { (Batch B) }\end{array}$ & $\begin{array}{c}\text { WBC } \\
(\mathbf{x 1 0 3} \text { cell } / \mu L)\end{array}$ & Lymphocytes (\%) & $\begin{array}{c}\text { Platelet } \\
(\mathbf{x 1 0 3} \text { cell } / \mu \mathrm{L})\end{array}$ & $\begin{array}{c}\text { PDW } \\
\text { (fL) }\end{array}$ & $\begin{array}{r}\text { MPV } \\
(\mathbf{f L})\end{array}$ & P-LCR (\%) \\
\hline Control & $11.98 \pm 0.76$ & $78.52 \pm 2.20$ & $938.80 \pm 24.38$ & $10.90 \pm 0.51$ & $9.10 \pm 0.41$ & $17.76 \pm 1.25$ \\
\hline Sildenafil & $8.26 \pm 0.79 c$ & $81.22 \pm 6.33$ & $864.00 \pm 22.13$ & $9.44 \pm 0.40$ & $8.04 \pm 0.22 \mathrm{c}$ & $11.96 \pm 1.31 \mathrm{c}$ \\
\hline Tadalafil & $10.78 \pm 1.65$ & $75.90 \pm 2.73$ & $845.80 \pm 45.72$ & $10.79 \pm 0.28$ & $8.24 \pm 0.25$ & $15.62 \pm 1.09$ \\
\hline Tramadol & $8.52 \pm 0.26 \mathbf{c}$ & $85.92 \pm 3.59$ & $865.40 \pm 31.08$ & $11.26 \pm .83 \mathbf{d}$ & $8.60 \pm 0.24$ & $16.14 \pm 1.98$ \\
\hline $\mathbf{S}+\mathbf{T}$ & $12.36 \pm 0.88 \mathbf{d}, \mathbf{i}$ & $83.16 \pm 4.58$ & $933.00 \pm 58.66$ & $10.92 \pm 0.39$ & $8.78 \pm 0.33$ & $17.36 \pm 1.80 \mathrm{~d}$ \\
\hline
\end{tabular}

Values are expressed as mean \pm SEM, $n=5 . c=p<0.05$ vs control; $d=p<0.05$ vs sildenafil; $i=p<0.05$ vs tramadol.

Table 7: Comparison of Haematological Indices between the treated and recovery group of animals.

\begin{tabular}{|c|c|c|c|c|c|c|c|c|c|c|}
\hline \multirow[t]{2}{*}{ Parameter } & \multicolumn{5}{|c|}{8 Weeks Treatment (Batch A) } & \multicolumn{5}{|c|}{8 Weeks Recovery (Batch B) } \\
\hline & Control & Sildenafil & Tadalafil & Tramadol & $\mathbf{S}+\mathbf{T}$ & Control & Sildenafil & Tadalafil & Tramadol & $\mathbf{S}+\mathbf{T}$ \\
\hline $\mathrm{RBC}(\mathrm{x106}$ cell/ $\mu \mathrm{L})$ & $7.32 \pm 0.24$ & $5.55 \pm 0.49$ & $5.67 \pm 0.57$ & $4.80 \pm 0.19$ & $3.95 \pm 0.7$ & $7.71 \pm 0.16$ & $7.86 \pm 0.17 \mathbf{b}$ & $7.30 \pm 0.27 \mathrm{c}$ & $7.35 \pm 0.34 \mathbf{a}$ & $7.63 \pm 0.05 \mathbf{a}$ \\
\hline $\mathrm{Hb}(\mathrm{g} / \mathrm{dL})$ & $14.20 \pm 0.50$ & $10.57 \pm 0.43$ & $13.94 \pm 0.21$ & $10.52 \pm 0.46$ & $9.76 \pm 0.89$ & $14.74 \pm 0.17$ & $14.80 \pm 0.28 \mathbf{a}$ & $13.84 \pm 0.33$ & $13.66 \pm 0.65 \mathbf{b}$ & $14.46 \pm 0.70 \mathbf{b}$ \\
\hline PCV (\%) & $44.40 \pm 1.41$ & $37.34 \pm 0.46$ & $41.48 \pm 0.66$ & $38.38 \pm 0.39$ & $35.56 \pm 1.04$ & $45.58 \pm 0.81$ & $46.70 \pm 1.32 \mathbf{a}$ & $44.40 \pm 1.09$ & $43.14 \pm 1.90 \mathbf{c}$ & $44.34 \pm 0.81 \mathbf{a}$ \\
\hline MCV (fL) & $61.44 \pm 1.26$ & $51.16 \pm 1.02$ & $57.92 \pm 0.69$ & $41.60 \pm 0.90$ & $40.34 \pm 0.89$ & $61.36 \pm 0.99$ & $59.36 \pm 0.56 \mathbf{b}$ & $58.16 \pm 0.65$ & $58.76 \pm 0.80 \mathbf{a}$ & $59.06 \pm 0.79 \mathbf{a}$ \\
\hline MCH (pg) & $19.62 \pm 0.37$ & $11.06 \pm 0.45$ & $13.74 \pm 0.45$ & $10.20 \pm 0.47$ & $10.56 \pm 0.52$ & $20.02 \pm 0.68$ & $18.84 \pm 0.13 \mathbf{a}$ & $17.96 \pm 0.33 \mathbf{a}$ & $18.60 \pm 0.22 \mathbf{a}$ & $18.34 \pm 0.20 \mathbf{a}$ \\
\hline MCHC (g/dL) & $31.68 \pm 0.22$ & $21.92 \pm 0.25$ & $25.66 \pm 0.71$ & $21.52 \pm 0.74$ & $19.88 \pm 0.42$ & $31.86 \pm 0.58$ & $31.72 \pm 0.34 \mathbf{a}$ & $31.56 \pm 0.50 \mathbf{a}$ & $31.62 \pm 0.16 \mathbf{a}$ & $30.68 \pm 0.47 \mathbf{a}$ \\
\hline RDW-SD (fL) & $33.56 \pm 0.74$ & $29.96 \pm 0.49$ & $30.22 \pm 0.82$ & $26.34 \pm 0.77$ & $21.42 \pm 0.16$ & $34.00 \pm 0.53$ & $33.46 \pm 0.55 \mathbf{b}$ & $34.80 \pm 0.71 \mathbf{b}$ & $36.48 \pm 2.26 \mathbf{b}$ & $35.08 \pm 1.48 \mathbf{a}$ \\
\hline RDW-CV (\%) & $13.82 \pm 0.68$ & $12.24 \pm 0.46$ & $12.72 \pm 0.69$ & $12.62 \pm 0.37$ & $9.80 \pm 0.12$ & $14.72 \pm 0.86$ & $15.16 \pm 0.46 \mathbf{b}$ & $16.08 \pm 0.69$ & $17.14 \pm 1.33$ & $16.22 \pm 0.54 \mathbf{a}$ \\
\hline $\mathrm{WBC}(\times 103 \mathrm{cell} / \mu \mathrm{L})$ & $11.74 \pm 0.76$ & $5.30 \pm 0.46$ & $7.93 \pm 0.38$ & $8.52 \pm 0.26$ & $6.50 \pm 0.32$ & $11.98 \pm 0.76$ & $8.26 \pm 0.79 c$ & $10.78 \pm 1.65$ & $8.52 \pm 0.26$ & $12.36 \pm 0.88 \mathbf{a}$ \\
\hline Lymphocytes (\%) & $74.68 \pm 2.69$ & $71.00 \pm 0.49$ & $79.74 \pm 4.04$ & $72.64 \pm 1.05$ & $62.48 \pm 0.61$ & $78.52 \pm 2.20$ & $81.22 \pm 6.33$ & $75.90 \pm 2.73$ & $85.92 \pm 3.59 c$ & $83.16 \pm 4.58 \mathbf{b}$ \\
\hline $\begin{array}{l}\text { Platelet }(\times 103 \\
\text { cell } / \mu \mathrm{L})\end{array}$ & $936.00 \pm 14.55$ & $700.40 \pm 7.83$ & $686.60 \pm 15.13$ & $762.20 \pm 33.55$ & $713.60 \pm 0.83$ & $938.80 \pm 24.38$ & $864.00 \pm 22.13 \mathbf{a}$ & $845.80 \pm 45.72 \mathrm{c}$ & $865.40 \pm 31.08$ & $933.00 \pm 58.66 \mathbf{b}$ \\
\hline PDW (fL) & $11.50 \pm 0.80$ & $9.92 \pm 0.44$ & $10.28 \pm 0.33$ & $9.44 \pm 0.07$ & $9.86 \pm 0.18$ & $10.90 \pm 0.51$ & $9.44 \pm 0.40$ & $10.79 \pm 0.28$ & $11.26 \pm 0.83$ & $10.92 \pm 0.39$ \\
\hline MPV (fL) & $9.10 \pm 0.41$ & $8.16 \pm 0.17$ & $8.26 \pm 0.15$ & $7.76 \pm 0.12$ & $8.18 \pm 0.07$ & $9.10 \pm 0.41$ & $8.04 \pm 0.22$ & $8.24 \pm 0.25$ & $8.60 \pm 0.24 c$ & $8.78 \pm 0.33$ \\
\hline P-LCR (\%) & $18.06 \pm 2.89$ & $12.44 \pm 1.28$ & $11.88 \pm 0.58$ & $10.16 \pm 0.75$ & $12.66 \pm 0.57$ & $17.76 \pm 1.25$ & $11.96 \pm 1.31$ & $15.62 \pm 1.09 \mathrm{c}$ & $16.14 \pm 1.98 \mathbf{c}$ & $17.36 \pm 1.80$ \\
\hline
\end{tabular}

Values are mean \pm SEM, $n=5 . a=p<0.001, b=p<0.01, c=p<0.05$ vs batch $A$

\section{WBC counts and platelet indices}

Table 6 shows the results for WBC count $\left(\times 10^{3}\right.$ cell $\left./ \mu \mathrm{L}\right)$ and platelet indices for control, sildenafil, tadalafil, tramadol and $\mathrm{S}$ $+\mathrm{T}$ groups after withdrawal of treatment. WBC count was significantly $(\mathrm{p}<0.05)$ lower in sildenafil and tramadol groups compared with control but significantly $(\mathrm{p}<0.05)$ higher in $\mathrm{S}+\mathrm{T}$ group compared with sildenafil and tramadol groups. There was no significant difference in lymphocytes count and platelet count $\left(\mathrm{x} 10^{3} \mathrm{cell} / \mu \mathrm{L}\right)$ in all groups.

The only significant difference in PDW (fL) was an increase $(\mathrm{p}<0.05)$ in PDW in tramadol group compared with sildenafil group. MPV (fL) was significantly $(\mathrm{p}<0.05)$ lower in sildenafil group compared with control. P-LCR (\%) was significantly lower $(\mathrm{p}<0.05)$ in sildenafil group compared with control and higher $(\mathrm{p}<0.05)$ in $\mathrm{S}+\mathrm{T}$ group compared with sildenafil group.

\section{Comparison of haematological indices between the treated and recovery groups}

\section{Red blood cell (RBC) count, Haemoglobin (Hb) concentration and packed cell volume}

Table 7 shows the results for $\mathrm{RBC}$ count $\left(\times 10^{6}\right.$ cell $\left./ \mu \mathrm{L}\right)$, haemoglobin concentration $(\mathrm{g} / \mathrm{dL})$ and packed cell volume $(\%)$ in the treated (Batch A) and recovery (Batch B) groups. RBC count was significantly higher in sildenafil $(p<0.01)$, tadalafil $(p<0.05)$, tramadol $(\mathrm{p}<0.001)$ and $\mathrm{S}+\mathrm{T}(\mathrm{p}<0.001)$ recovery groups compared with their corresponding batch A groups. Sildenafil recovery group had a significantly $(\mathrm{p}<0.001)$ higher $\mathrm{Hb}$ concentration $(\mathrm{g} / \mathrm{dL})$ compared with its batch $\mathrm{A}$ group. $\mathrm{Hb}$ concentration was also significantly $(\mathrm{p}<0.01)$ higher in tramadol and $\mathrm{S}+\mathrm{T}$ recovery groups compared with the corresponding batch A groups. PCV (\%) was significantly higher in sildenafil
( $\mathrm{p}<0.001)$, tramadol $(\mathrm{p}<0.05)$ and $\mathrm{S}+\mathrm{T}(\mathrm{p}<0.001)$ recovery groups compared with the corresponding treated groups.

\section{Red blood cell absolute values}

Results for red blood cell absolute values in the treated and recovery groups are presented in table 7. MCV (fL) was significantly higher in sildenafil $(\mathrm{p}<0.01)$, tramadol $(\mathrm{p}<0.001)$ and $\mathrm{S}+\mathrm{T}(\mathrm{p}<0.001)$ recovery groups compared with the corresponding treated groups. $\mathrm{MCH}(\mathrm{pg})$ and $\mathrm{MCHC}(\mathrm{g} / \mathrm{dL})$ were significantly $(\mathrm{p}<0.001)$ higher in all recovery groups compared with the treated groups. RDW-SD (fL) was also significantly higher in sildenafil $(\mathrm{p}<0.01)$, tadalafil $(\mathrm{p}<0.01)$, tramadol $(\mathrm{p}<0.01)$ and $\mathrm{S}+\mathrm{T}$ $(\mathrm{p}<0.001)$ recovery groups compared with the corresponding treated groups. RDW-CV was significantly higher in sildenafil $(\mathrm{p}<0.01)$ and $\mathrm{S}+\mathrm{T}(\mathrm{p}<0.001)$ recovery groups in comparison with the treated groups.

\section{WBC count and platelet indices}

WBC count $\left(\times 10^{3}\right.$ cells $\left./ \mu \mathrm{L}\right)$ was significantly higher in sildenafil $(\mathrm{p}<0.05)$ and $\mathrm{S}+\mathrm{T}(\mathrm{p}<0.001)$ recovery groups compared with the treated groups. Lymphocytes count $(\%)$ was significantly higher in tramadol $(\mathrm{p}<0.05)$ and $\mathrm{S}+\mathrm{T}(\mathrm{p}<0.01)$ recovery groups compared with the treated groups. Platelets count $\left(\times 10^{3}\right.$ cells $\left./ \mu \mathrm{L}\right)$ was also significantly higher in sildenafil $(\mathrm{p}<0.001)$, tadalafil $(\mathrm{p}<0.05)$ and $\mathrm{S}+\mathrm{T}(\mathrm{p}<0.01)$ recovery groups compared with their corresponding treated groups. There was no significant difference in PDW (fL) and MPV (fL) in the recovery groups compared with the treated groups except for MPV which was significantly $(\mathrm{p}<0.05)$ higher in the tramadol recovery group compared with its treated group. P-LCR $(\%)$ was significantly higher $(\mathrm{p}<0.05)$ in tadalafil and tramadol recovery groups compared with the corresponding treated groups (Table 7). 


\section{DISCUSSION}

PDE5i are used for the treatment of erectile dysfunction. Tramadol is an opioid used for pain relief and treatment of premature ejaculation. Both drugs have been administered in combination for the above reasons. Both classes of drugs are associated with various adverse effects. This study evaluated possible reversal of alterations in blood parameters after treatment with sildenafil, tadalafil, tramadol and sildenafil + tramadol and subsequent withdrawal of treatment in rats.

$\mathrm{RBC}$ count, $\mathrm{Hb}$ concentration and PCV were significantly decreased in all treated groups compared with the control. The decrease in $\mathrm{Hb}$ concentration in tadalafil group was not significant compared with the control. These results are in tandem with the findings of Aldalou et al. (2014) and Elyazji et al. (2013) that showed a decrease in RBC count and $\mathrm{Hb}$ concentration following combined administration of sildenafil and tramadol and tramadol administration only, respectively, in domestic rabbits. It is also likely that sildenafil, tadalafil and tramadol have inhibitory effects on erythropoiesis. PCV was decreased following the decreased $\mathrm{RBC}$ count. The decreased $\mathrm{Hb}$ concentration may be due to the decrease in $\mathrm{RBC}$ count or impairment of heme biosynthesis during the process of erythropoiesis.

$\mathrm{MCV}, \mathrm{MCH}, \mathrm{MCHC}$ and RDW-SD were significantly decreased in the treated groups compared with the control. The reduction were more in the $\mathrm{S}+\mathrm{T}$ group. RDW-CV was significantly lowered in $\mathrm{S}+\mathrm{T}$ group compared with the control, but was not significantly different in other treated groups compared with the control. Our findings contradicts the findings of Aldalou et al. (2014) and Elyazji et al. (2013)who both reported that combined administration of sildenafil and tramadol, and tramadol administration alone, respectively, significantly increased MCV and MCH but agrees with their results for decreased MCHC. Decreased MCV indicates that the RBCs became microcytic following administration of the drugs. The decreased $\mathrm{MCH}$ and $\mathrm{MCHC}$ are in line with the decreased $\mathrm{Hb}$ concentration which indicates that administration of sildenafil, tadalafil, tramadol and sildenafil + tramadolmay inhibit biosynthesis of heme in the bone marrow. Diminished $\mathrm{MCH}$ is observed in hypochromicanaemia. It is likely that the administered drugs suppressed the synthesis of iron which resulted in the presence of microcytic RBCs and hence, decreased $\mathrm{Hb}$ concentration. The lower values of RDW-SD indicates small variation in the sizes of circulating RBCs.

WBC count was significantly decreased in allthe treated groups compared with the control. Lymphocytes count was only significantly decreased in the $\mathrm{S}+\mathrm{T}$ group, compared with the control, although non-significant reductions were observed in the sildenafil and tramadol groups. The significant decrease in WBC count indicates the suppression of defense mechanism and immune system of rats. Platelets count, PDW, MPV and P-LCR were significantly decreased in all the treated groups, compared with the control. This indicates adverse effect on the body's clotting mechanism caused by the various drugs administered. Our results contradict the findings of Shatha and Adnan (2015) on blood homeostasis who concluded that, PDE5i (sildenafil \&tadalafil) increased platelets activity and activated their aggregation. Our result for decreased platelets count is consistent with that ofAldalou et al. (2014).

When the recovery groups were compared with their respective treated groups, $\mathrm{RBC}$ count, $\mathrm{Hb}$ concentration and $\mathrm{PCV}$ were significantly higher in the recovery groups, compared with the treated groups (Table 7). RBC count was significantly increased in the tadalafil recovery group compared with its treated group, but its $\mathrm{Hb}$ concentration and PCV were not significantly changed. This shows that tadalafil caused more severe alterations in $\mathrm{Hb}$ concentration and PCV than sildenafil and tramadol since tadalafil group was the only group that exhibited poor reversibility of $\mathrm{Hb}$ concentration and $\mathrm{PCV}$.

$\mathrm{MCV}, \mathrm{MCH}, \mathrm{MCHC}, \mathrm{RDW}-\mathrm{SD}$ and RDW-CV increased in all recovery groups following withdrawal of treatment. Although MCV and RDW-CV in tadalafil recovery group and RDW-CV in tramadol recovery group were not significantly increased, all other red cell absolute values were significantly higher in the recovery groups compared with the treated groups. The higher values of RDW-CV observed in the $\mathrm{S}+\mathrm{T}$ recovery group is due to the effect of sildenafil, since tramadol recovery group alone did not show significant difference, indicating that, tadalafil and tramadol exerted greater effects on $\mathrm{Hb}$ concentration than on count and size of circulating RBCs. However, there was strong reversibility of red cell absolute values exhibited by the different drugs, but tadalafil poorly reversed MCV and RDW-SD values.

WBCs count was significantly lower in sildenafil and tramadol groups, but lymphocytes count was not significantly altered in all groups following withdrawal of treatment (Table 6). When treated and recovery groups were compared, WBCs count was significantly higher in sildenafil and $\mathrm{S}+\mathrm{T}$ recovery groups, and lymphocytes count significantly higher in tramadol and $\mathrm{S}+\mathrm{T}$ recovery groups, compared with their respective treated groups. This shows that sildenafil and tramadol administered together exhibited strong reversibility of alterations in WBCs and lymphocytes counts.

MPV and P-LCR were significantly lowered in sildenafil group compared with the control. Other groups showed no significant difference in platelets indices compared with the control following withdrawal of treatment (Table 6). When recovery groups were compared with treated groups, platelets count was found to be significantly higher in sildenafil, tadalafil and $\mathrm{S}+\mathrm{T}$ recovery groups, compared with their respective treated groups. The significant increase in platelets count caused by sildenafil+tramadol is due to the effect of sildenafil since tramadol alone did not show significant difference. There was however strong reversibility of platelets count exhibited by sildenafil and tadalafil.

MPV was significantly higher in tramadol recovery group and P-LCR significantly higher in tadalafil and tramadol recovery groups, compared with their respective treated groups, implying that, the alterations in platelets size caused by tramadol 
was however, strongly reversed following withdrawal of treatment.

\section{CONCLUSION}

The recreational use of sildenafil, tadalafil, tramadol and sildenafil+tramadol causes alterations in haematological parameters, with appreciable reversibility following their withdrawal. However, platelet indices were poorly reversed in sildenafil and tramadol recovery groups. Thus, patients with bleeding disorders or blood coagulation disorders may be at risk of worsening their condition with high doses of these drugs. Although haematological alterations were reversed following withdrawal of treatment with sildenafil, tadalafil, tramadol and sildenafil + tramadol, it is important for anaemic patients to refrain from high doses of these drugs to avoid worsening their condition.

\section{CONFLICT OF INTEREST: None declared.}

\section{REFERENCES}

Akhtardanesh, B., Sharifi, H., Rasodi, R. and Aghazamani, M. Evaluation of haematological and biochemical changes after short term tramadol usage in healthy dogs. Iranian Journal of Veterinary Medicine, 2014; 8 (1): 41-45.

Aldalou, A.R., Abdel-Aziz,I. and Shahwan, O. Impact of giving sildenafil (viagra) / tramadol (tramal) combination on the blood of domestic rabbits. Journal of Science, 2014; 4 (3): 162-169.

Aversa, A., Bruzziches, R., Pili, M. and Spera, G. Phosphodiesterase 5 inhibitors in the treatment of erectile dysfunction. Curr Pharm Des. 2006; 12: 3467- 3484.

Bamigbade, T.A., Davidson, C., Langford, R.M. and Stamford, J.A. Actions of tramadol, its enantiomers and principal metabolite, odesmethyltramadol,on serotonin (5-ht) efflux and uptake in the rat dorsalraphe nucleus. Br J Anaesth, 1997; 79: 352-356.

Eherer, A.J., Schwetz, I., Hammer, H.F., Petnehazy, T., Scheidl, S.J., Weber, K. and Krejs, G.J. Effect of sildenafil on oesophageal motor function in healthy subjects and patients with oesophageal motor disorders. Gut, 2002; 50: 758-764.

Elyazji, N. R., I. Abdel-Aziz, A. Aldalou, and O. Shahwan. The effects of tramadol hydrochloride administration on the hematological and biochemical profiles of domestic male rabbits.IUG J Natural and Eng Studies, 2013; 21: 51-65.

Goldberg, D.J., French, B., McBride, M.G., Marino, B.S. and Mirarchi, N. Impact of oral sildenafil on exercise performance in children and young adults after the fontan operation: A randomized, double-blind, placebo-controlled, crossover trial. Circulation, 2011; 123: 1185-1193.

Grond, S. and Sablotzki, A. Clinical pharmacology of tramadol. Clin. Pharmacokinet, 2004;43: 879-923.

Kyratsas, C.C. Dalla, E., Anderzhanova, A., Polissidis, N., Kokras, K.K. and Daifoti, P.Z. Experimental evidence for sildenafil's action in the central nervous system: Dopamine and serotonin changes in the medial preoptic area and nucleus accumbens during sexual arousal. $J$. Sexual Med., 2013; 10: 719-729.

Lauver, D.A., Carey, E.G., Bergin, I.L., Lucchesi, B.R. and Gurm, H.S. Sildenafil citrate for prophylaxis of nephropathy in an animal model of contrast-induced acute kidney injury. PLOS ONE, 2014; Vol. 9. 10.1371/journal.pone.0113598

Nna, V.U., Akpan, U.P. and Osim, E.E. Separately administered phosphodiesterase-5 inhibitors (sildenafil and tadalafil) and opioid (tramadol), reversibly alter serum lipid profile in male albino wistar rats. Asian J. Biochem. 2015a; 10: 132-144.
Nna, V.U., Akpan, U.P., Okon, V.E. and Atangwho, I.J. Hepatotoxicity following separate administration of two phosphodiesterase-5 inhibitors (sildenafil \&tadalafil) and opioid (tramadol); evaluation of possible reversal following their withdrawal. Journal of Applied Pharmaceutical Science, 2015b;5 (08), 105-113.

Nna, V.U.,Ani, E.J., Ofutet, E.O., Ofem, O.E., Iroh, C.E. and Osim, E.E. Recurrent side effects following chronic recreational use of sexual stimulants among male subjects in Calabar, Cross River State, Nigeria. Der Pharmacia Lettre, 2014a; 6 (6): 56-61.

Nna, V.U., Ofem, O.E. and Osim, E.E. Prevalence of sexual stimulant abuse unrelated to presence of erectile dysfunction among male subjects in Calabar, Cross River State, Nigeria. International Union of Physiological Sciences (IUPS)/34th Annual Scientific Conference of the Physiological Society of Nigeria, 20 - 21 October, 2014b.

Oka, V.O., Udefa, A.L., Nna, V.U. and Owu, D.U. Sildenafil citrate and tramadol administered separately and in combination affects basal metabolic rate, triiodothyronine (T3) and cortisol levels in albino wistar rats. Trends in Medical Research, 2015;10 (3): 51-62.

Safarinejad, M.R. and Hosseini, S.Y. Safety and efficacy of tramadol in the treatmentof premature ejaculation: A double-blind, placebo-controlled, fixed-dose, randomized study.J. Clin. Psychopharmacol, 2006; 26: 27-31.

Salam, A.R., Rahman, A., Sultana, U.N. and Sultan, Z. Study of in vitro interaction of sildenafil citrate with bovine serum albumin by fluorescence spectroscopy.Pharmacol. Pharm, 2015; 6: 94-101.

Salem, E.A., Wilson, S.K., Bissada, N.K., Delk, J.R., Hellstrom, W.J. and Cleves, M.A. Tramadol HCL has promise in on-demand use to treat premature ejaculation. J. Sexual Med., 2008; 5: 188-193.

Shatha, H.A. and Adnan, M. The Effect of Pde-5 Inhibitors on blood homeostasis in relation to the type \& duration of therapy. I.J.S.N 2015;.6 (1):28-35.

Shinlapawittayatorn, K., Chattipakorn, S. and Chattipakorn, N. Effect of sildenafil citrate on the cardiovascular system. Braz. J. Med. Biol. Res, 2005; 38: 1303-1311.

Supuran, C.T., Mastrolorenzo, A., Barbaro, G. and Scozzafava, A. Phosphodiesterase 5 inhibitors-drug design and differentiation based on selectivity, pharmacokinetic and efficacy profiles. Curr Pharm Des. 2006; 12: 3459-3465.

Tas, A., Karasu, A., Comba, B., Aksu, D.S., Duz, E. and Tanritanir, P. Effects of Sildenafil citrate on the haematological parameters in early phase of wound healing in diabetic rats. Asian Journal of Animal and Verterinary Advances, 2011; 6 (3):290-296.

Yildiz, H., Durmuş, A.S., Şimşek, H. and Yaman, I. Effects of sildenafil citrate on torsion/detorsion-induced changes in red blood cell and plasma lipid peroxidation, antioxidants, and blood hematology of male rats. European Journal of Obstetrics \&Gynecology and Reproductive Biology, 2011; 159 (2):359-363.

Zusman, R.M., Prisant, L.M., Brown, M.J. and Sildenafil Study Group. Effect of sildenafil citrate on blood pressure and heart rate in men with erectile dysfunction taking concomitant antihypertensive medication. J. Hypertens, 2000; 18: 1865-1869.

\section{How to cite this article:}

Victor U. Nna, Victor O. Oka, Augustine L. Udefa, Emmanuel O. Ofutet, Ofem E. Ofem. High Doses of PDE5 inhibitors and tramadol reversibly alters haematological parameters in rats. J App Pharm Sci, 2016; 6 (04): 086-092. 